Submitted to the Astrophysical Journal

Preprint typeset using LATEX style emulateapj v. 05/04/06

\title{
THE X-RAY CAVITIES, FILAMENTS AND COLD FRONTS IN THE CORE OF THE GALAXY GROUP
} NGC 5044

\author{
Fabio Gastaldello ${ }^{1,2,3}$, David A. Buote ${ }^{3}$, Pasquale Temi ${ }^{4,5}$, Fabrizio Brighenti $^{6,7}$, William G. Mathews ${ }^{7}$ \& Stefano \\ ETTORI $^{8,9}$ \\ Submitted to the Astrophysical Journal
}

\begin{abstract}
We present a two-dimensional analysis of the bright nearby galaxy group NGC 5044 using the currently available Chandra and $X M M$ data. In the inner $10 \mathrm{kpc}$ a pair of cavities are evident together with a set of bright $\mathrm{X}$-ray filaments. If the cavities are interpreted as gas displaced by relativistic plasma inflated by an AGN, even in the absence of extended $1.4 \mathrm{GHz}$ emission, this would be consistent with a recent outburst as also indicated by the extent of dust and $\mathrm{H} \alpha$ emission. The soft X-ray filaments coincident with $\mathrm{H} \alpha$ and dust emission are cooler than the ones which do not correlate with optical and infrared emission. We suggest that dust-aided cooling contributes to form warm $\left(T \sim 10^{4} \mathrm{~K}\right)$ gas, emitting $\mathrm{H} \alpha$ radiation. At $31 \mathrm{kpc}$ and $67 \mathrm{kpc}$ a pair of cold fronts are present, indicative of sloshing due to a dynamical perturbation caused by accretion of a less massive group, also suggested by the peculiar velocity of the brightest galaxy NGC 5044 with respect to the mean group velocity.

Subject headings: cooling flows — galaxies: clusters: general — galaxies: clusters: individual

(NGC 5044) — X-rays: galaxies: clusters
\end{abstract}

\section{INTRODUCTION}

The current X-ray observatories, Chandra and XMM, have revolutionized our understanding of the cores of relaxed galaxies, groups and clusters, which show a highly peaked $\mathrm{X}$ ray emission from a hot interstellar medium whose radiative cooling time is less than $1 \mathrm{Gyr}$ (for recent determinations of cooling times in these objects see for example Voigt \& Fabian 2004; Sanderson et al. 2006; Jetha et al. 2007). In the absence of heating, a cooling flow is established, in which the gas cools, condenses and flows toward the center, accreting onto the central galaxy (Fabian 1994). However the mass sink for all this supposedly cooling and condensing gas has never been found (Donahue \& Voit 2004). X-ray observations with Chandra and $X M M$ have established that there is little evidence for emission from gas cooling below $\sim T_{\text {vir }} / 3$ : just when gas should be cooling most rapidly it appears not to be cooling at all (see the recent review by Peterson \& Fabian 2006, and references therein).

A compensating heat source must therefore resupply the radiative losses, and many possibilities have been proposed, including thermal conduction (e.g., Narayan \& Medvedev 2001), energy released by mergers (e.g., Motl et al. 2004) or by supernovae (e.g., Silk et al. 1986). However, feedback from the central AGN has become the most appealing solution

\footnotetext{
${ }^{1}$ IASF-Milano, INAF, via Bassini 15, Milano 20133, Italy

2 Occhialini Fellow

${ }^{3}$ Department of Physics and Astronomy, University of California at Irvine, 4129 Frederick Reines Hall, Irvine, CA 92697-4575

${ }^{4}$ Astrophysics Branch, NASA/Ames Research Center, MS 245-6, Moffet Field, CA 94035

${ }^{5}$ SETI Institute, Mountain View, CA 94043; and Department of physics and Astronomy, University of Western Ontario, London ON N6A, 3K7, Canada

${ }^{6}$ Dipartimento di Astronomia, Università di Bologna, via Ranzani 1, Bologna 40127, Italy

7 UCO/Lick Observatory, University of California at Santa Cruz, 1156 High Street, Santa Cruz, CA 95064

${ }^{8}$ INAF, Osservatorio Astronomico di Bologna, via Ranzani 1, Bologna 40127, Italy

${ }^{9}$ INFN, Sezione di Bologna, viale Berti Pichat 6/2, I-40127 Bologna, Italy
}

to the problem (see the recent review by McNamara \& Nulsen 2007, and references therein). There is, in fact, clear observational evidence for AGN heating as the majority of brightest central galaxies of clusters and groups host a radio loud AGN (e.g., Burns 1990; Best et al. 2007) and, following the launch of Chandra, in an increasing number of objects such disturbances as shocks, ripples and cavities have been found in the central atmospheres of clusters, groups and elliptical galaxies (e.g., Fabian et al. 2006; Bîrzan et al. 2004; Dunn \& Fabian 2006; Forman et al. 2005; Vrtilek et al. 2002; Jetha et al. 2007; Croston et al. 2008; Finoguenov \& Jones 2001; Allen et al. 2006). The cavities, which appear as X-ray surface brightness depressions, have been interpreted as bubbles of low density relativistic plasma inflated by radio jets, displacing the thermal gas causing $P d V$ heating (e.g., Churazov et al. 2002), although Mathews \& Brighenti (2008) pointed out that cavity formation contributes thermal energy that may offset radiative cooling only by injecting ultra-hot but non relativistic gas; X-ray cavities formed solely from relativistic gas have a global cooling effect. In most cases, the energy introduced by the AGN is more than sufficient to counteract putative cooling flows (McNamara \& Nulsen 2007), although the physical process of the coupling of the feedback energy with the ambient medium is not well understood. While many of the observed X-ray cavities are filled with plasma emitting in the radio at $1.4 \mathrm{GHz}$, some are undetected at this frequency and have been referred to as "ghost cavities". These may result from the aging of the relativistic particle population and be the signature of a previous AGN outburst. Observations at low radio frequency of several rich clusters with ghost cavities show that they are indeed filled with relativistic plasma (e.g., A 2597, Clarke et al. 2005), although even low frequency emission cannot be easily detected.

However, some net cooling is probably occurring, as the galaxies at the center of cool cores show properties not shared by typical elliptical galaxies (Crawford 2004): 1) the presence of strong, low-ionization emission line nebulae both in clusters (e.g., Crawford et al. 1999; Conselice et al. 2001) and in groups (e.g. Macchetto et al. 1996); 2) the galaxies 
at the center of clusters that display these nebular emission show a component of excess ultraviolet/blue continuum associated with young stars (e.g., Cardiel et al. 1998; Crawford et al. 1999). This excess blue light is again extended on scales of several kpc and there is evidence that some (spatially extended) star formation has been triggered by interaction with the radio source (McNamara 2004) 3) CO emission lines have been detected in several cool core clusters at millimeter wavelengths (e.g., Edge 2001; Salomé \& Combes 2003), implying the presence of a substantial amount of warm molecular gas $\left(10^{9-11.5} \mathrm{M}_{\odot}\right)$ within a $50 \mathrm{kpc}$ radius of the central galaxy. Less massive warmer molecular regions have been observed in $\mathrm{H}_{2}$ lines, which are often spatially associated with the stellar UV and $\mathrm{H} \alpha$ emission extending over $\sim 20 \mathrm{kpc}$ (Jaffe et al. 2005).

The origin and excitation mechanism of the nebular $\mathrm{H} \alpha$ emission have been much debated and they are still poorly known. The nebulae require a constant and distributed heating source (e.g. Johnstone \& Fabian 1988) which could be stellar (e.g. massive OB stars, Allen et al. 1992) or the ICM (e.g. conduction, Sparks et al. 1989). Deep Chandra X-ray imaging of Perseus (Fabian et al. 2003b) and M87 (Sparks et al. 2004) have renewed interest in conduction because of the spatial coincidence of filamentary $\mathrm{H} \alpha$ and soft X-ray emission, which can be due to conduction and mixing of the cold gas with the ICM. The disposition of some of the optical filaments in Perseus strongly suggests that the filaments are due to the buoyant radio bubbles drawing out the cold gas from a central reservoir (Fabian et al. 2003b) and it reveals the possibility of tracing the flow of the rising bubbles (Hatch et al. 2006). But the question is still open if galaxy-galaxy interactions are required to stimulate the central optical nebulae and if the optical filaments are trails of galaxies punching through a molecular hydrogen reservoir as suggested by the examples in Wilman et al. (2006). The excitement caused by the shift in the cooling flow paradigm is stimulating also a renewed theoretical interest in the quest for the origin and excitation of molecular (Ferland et al. 2008) and $\mathrm{H} \alpha$ emission (Nipoti \& Binney 2004; Pope et al. 2008; Revaz et al. 2008). In particular Nipoti \& Binney (2004) have shown how the $\mathrm{H} \alpha$ nebulae can be stable against thermal evaporation only for the conditions of temperature and pressure found in cool cores, explaining their association with that type of environment.

High resolution Chandra observations have also revealed another interesting and unexpected feature in the ICM of relaxed clusters: the presence of cold fronts. Many clusters have been found to exhibit sharp arc-shaped jumps in their gas density and temperature which, unlike shock fronts, have the gas on the dense side cooler, so the pressure is continuous across the front (e.g., Markevitch et al. 2000; Vikhlinin et al. 2001). In merging systems they were interpreted as contact discontinuities between gases from different sub-clusters (Markevitch et al. 2000). However cold fronts are present in the centers of many, if not most, relaxed clusters with cool cores (e.g., Mazzotta et al. 2001; Markevitch et al. 2003; Dupke \& White 2003; Ascasibar \& Markevitch 2006; Ghizzardi et al. 2007). The widely accepted scenario is that cold fronts in these systems are due to sloshing of the cool gas in the central gravitational potential, which is set off by minor mergers/accretions; the only necessary condition is a steep entropy profile as observed in relaxed clusters (Ascasibar \& Markevitch 2006).

Whereas a growing number of clusters and elliptical galaxies have deep enough multi-wavelength data (X-rays, radio and optical) to study the rich phenomenology of cool cores in a detailed spatially resolved fashion, only an handful of groups with such coverage exists, as for example the GEMS objects (Osmond \& Ponman 2004) presented in Rasmussen $\&$ Ponman (2007), and therefore, "unfortunately, AGN heating is not as well studied in groups as in clusters" (McNamara \& Nulsen 2007). Examination of AGN feedback at the mass scale of groups is valuable because, although the scale of outbursts in groups is less energetic and often on a smaller spatial scale than in clusters, the impact can be even more dramatic than in rich clusters due to the shallower group potential. Statistical studies examining the impact of AGN on groups are starting to address the points raised above (Croston et al. 2005; Jetha et al. 2007). Cold fronts have not been investigated in detail so far in relaxed groups of galaxies.

In this paper we show with more detail the currently available Chandra and XMM data for the galaxy group NGC 5044, one of the brightest groups in X-rays: it is one of the only 5 objects with $\mathrm{k} T<2 \mathrm{keV}$ in the HIFLUGCS sample (Reiprich $\&$ Böhringer 2002). Our estimate, using the new Chandra and $X M M$ data, for the bolometric (0.1-100 keV) X-ray luminosity within $r_{500}=443 h_{70}^{-1} \mathrm{kpc}$ (Gastaldello et al. 2007b) is $1.05 \pm 0.06 \times 10^{43} h_{70}^{-2} \mathrm{erg} \mathrm{s}^{-1}$, in good agreement with the ROSAT estimate presented in Reiprich \& Böhringer (2002) of $2.46 \times 10^{43} h_{50}^{-2}$ within $560 h_{50}^{-1} \mathrm{kpc}$. In our previous analysis, mainly focused on radial, azimuthally averaged properties of the system (Buote et al. 2003a,b), interesting features like holes and filaments within $10 \mathrm{kpc}$, a sharp edge, resembling a cold front, at $67 \mathrm{kpc}$, and unusually low iron abundances at large radii (Buote et al. 2004) were already pointed out. An $\mathrm{H} \alpha$ nebula is present in the core of NGC 5044 showing an extended filamentary structure (Goudfrooij et al. 1994a; Caon et al. 2000). NGC 5044 is also remarkable because Spitzer data show extended cold dust emitting at $70 \mu \mathrm{m}$ (Temi et al. 2007a). Moreover, Temi et al. (2007b) showed extended $8 \mu \mathrm{m}$ excess (likely arising from $\mathrm{PAH}$, polycyclic aromatic hydrocarbon, molecules) extending out to several kpc and spatially coincident with the $\mathrm{H} \alpha$ emitting nebulosity and the brightest soft X-ray emission. As proposed in Temi et al. (2007b), current evidence is consistent with an internal origin of this dust, which has been buoyantly transported from the galactic core out to several kpc into the hot X-ray emitting gas following an AGN outburst.

All distance-dependent quantities have been computed assuming $H_{0}=70 \mathrm{~km} \mathrm{~s}^{-1} \mathrm{Mpc}^{-1}, \Omega_{\mathrm{m}}=0.3$ and $\Omega_{\Lambda}=0.7$. At the redshift of $z=0.0091^{\prime}$ corresponds to $11.1 \mathrm{kpc}$. All the errors quoted are at the $68 \%$ confidence limit.

\section{OBSERVATIONS AND DATA PREPARATION}

NGC 5044 has been observed by Chandra with the ACIS-S configuration on September 1999 (obsID 798) for $20.7 \mathrm{ks}$ and by $X M M$ on January 2001 (obsID 0037950101) with the EPIC MOS and pn cameras for 23 and $20 \mathrm{ks}$, respectively (see also Buote et al. 2003a,b). We focused mainly on the Chandra data because its PSF is much better suited for the high-spatial resolution study that constitutes most of the paper. We take advantage of the $X M M$ data when we discuss the properties at larger radii (see section $\$ 3.2$ and $\S 4.2$ ) where the resolution requirements are not so important and we can benefit from the larger accessible field. Here we provide just a brief description of the data preparation, more details can be found in Gastaldello et al. (2007b).

\subsection{Chandra}




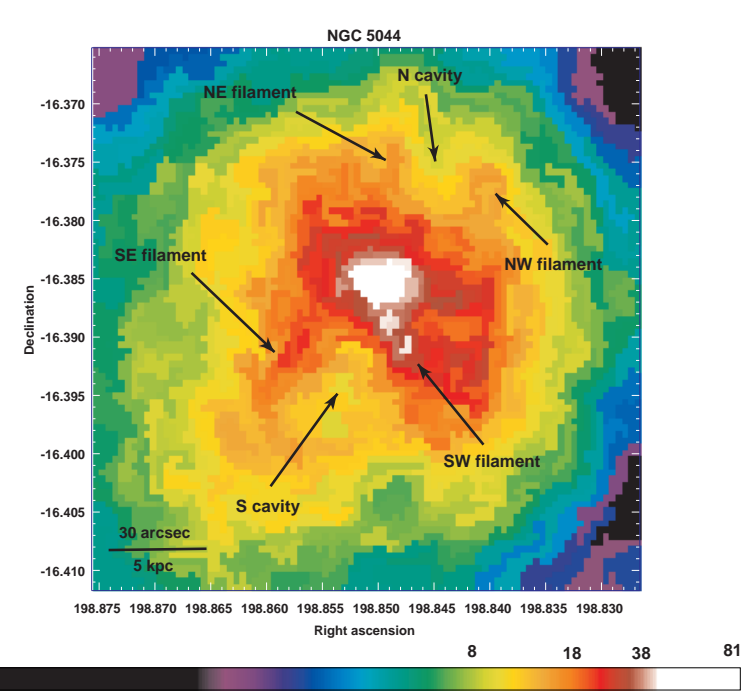

FIG. 1.- Chandra $0.5-5 \mathrm{keV}$ X-ray image of the inner $30 \times 30 \mathrm{kpc}$ of NGC 5044. The image has been processed to remove point sources, flat fielded with a $1.0 \mathrm{keV}$ exposure map. The figure has been contour binned using the algorithm of Sanders (2006) with a S/N of 10. Color bar units are total counts per pixel. Interesting features discussed in the text are highlighted.

The data were analyzed with the X-ray analysis packages CIAO 3.4 and Heasoft 6.4 in conjunction with the Chandra calibration database (Caldb) version 3.4.2. In order to ensure the most up-to-date calibration, all data were reprocessed from the "level 1" events files, following the standard Chandra data-reduction threads ${ }^{10}$. We applied the standard corrections to take account of a time-dependent drift in the detector gain and charge transfer inefficiency, as implemented in the CIAO tools. From low surface brightness regions of the active chips we extracted a light-curve (5.0-10.0 keV) to identify and excise periods of enhanced background. The observation was quiescent resulting in a final exposure time of 20 ks. Point source detection was performed using the CIAO tool wavdetect and removed, so as not to contaminate the diffuse emission, using appropriate elliptical regions containing $99 \%$ of their flux. We generated an image in the 0.5-5.0 $\mathrm{keV}$ and a corresponding exposure map computed at an energy of $1.0 \mathrm{keV}$. A zoom of the image in the inner region is presented in Fig.1 and Fig.10 and the the region covered by the ACIS-S3 chip is presented in Fig.4.

\section{2. $X M M$}

We generated calibrated event files with SAS v7.1.0 using the tasks emchain and epchain. We considered only event patterns $0-12$ for MOS and 0 for $\mathrm{pn}$, and the data were cleaned using the standard procedures for bright pixels and hot column removal and pn out-of-time correction. Periods of high background due to soft protons were filtered as in Gastaldello et al. (2007b) resulting in a net exposure time of $22 \mathrm{ks}$ for MOS1 and MOS2 and $17 \mathrm{ks}$ for pn. For each detector, we created images in the $0.5-2 \mathrm{keV}$ band with point sources detected using the task ewavelet and masked using circular regions of 25 radius centered at the source position. We created exposure maps for each detector and we combined the MOS images into a single exposure-corrected image, smoothed on a scale of $10^{\prime \prime}$, shown in Fig.5.

\footnotetext{
${ }^{10} \mathrm{http} / / / \mathrm{cxc}$. harvard.edu/ciao/threads/index.html
}

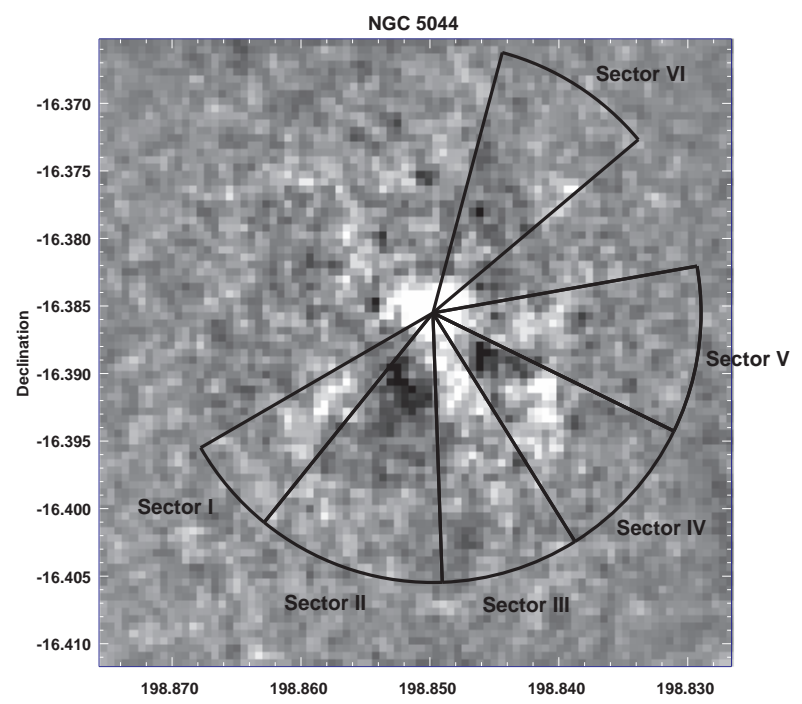

FIG. 2.- Unsharp-masked Chandra image created by subtracting a 0.5-5 $\mathrm{keV}$ intensity image smoothed by a $\sigma=10^{\prime \prime}$ Gaussian and one smoothed by a $\sigma=1^{\prime \prime}$ Gaussian. Areas in black show a deficit of counts, whereas areas in white show an excess of counts. Also shown are the angular sectors used for the surface brightness profiles shown in Fig.3, labeled by name according to the scheme of Table 1 .

\section{X-RAY IMAGES AND SURFACE BRIGHTNESS PROFILES}

\subsection{The inner $10 \mathrm{kpc}$}

The presence of a disturbed morphology with filamentary structure is already evident from the raw Chandra image and further confirmed by using the contour binning technique of Sanders (2006) (See Fig.1). Two depressions in surface brightness and multiple filamentary structures, some of them connected to the presence of the cavities, have been highlighted in Fig.1. To highlight the presence of structure we performed an "unsharp masking" of the Chandra image (e.g., Fabian et al. 2003a): the $0.5-5 \mathrm{keV}$ exposure-map-corrected image was smoothed with Gaussians of width 1 and 10 arcsec and the two smoothed images were then subtracted, with the resulting image shown in Fig.2. The southern cavity and the structure of filaments are clearly shown in the unsharp masked image. To quantify the observed structures we extracted from the exposure corrected image (the unsharp image was not used in the following analysis of the surface brightness profiles) an azimuthally averaged radial profile and profiles from selected angular sectors as depicted in Fig. 2 and listed in Table 1 (centered on the X-ray surface brightness peak coincident with the optical center of the galaxy NGC 5044 as listed by NED and by Macchetto et al. 1996, RA 13:15:24.0 and DEC -16 23 09, J2000 coordinates).

As it can be seen from Fig.3, there is a complex network of filamentary structure of enhanced emission which highlights dramatically regions of comparatively lower surface brightness. Two filaments, brighter and extended (in particular compared to the azimuthally averaged profile, also shown in the right panel of Fig.3), are evident in sector I (the SE filament) and sector III (the SW filament) with an embedded cavity in Sector II (also in this sector the emission is more extended compared to the average, i.e. there is an excess of counts for radii greater than $1^{\prime}$ ). The filament in sector III is brighter in the region $10^{\prime \prime}-30^{\prime \prime}$ but less extended, i.e. dimmer at $r>1^{\prime}$, than the overall emission at PA 120-182. The emission in sector IV can robustly be considered filamentary, with enhanced 

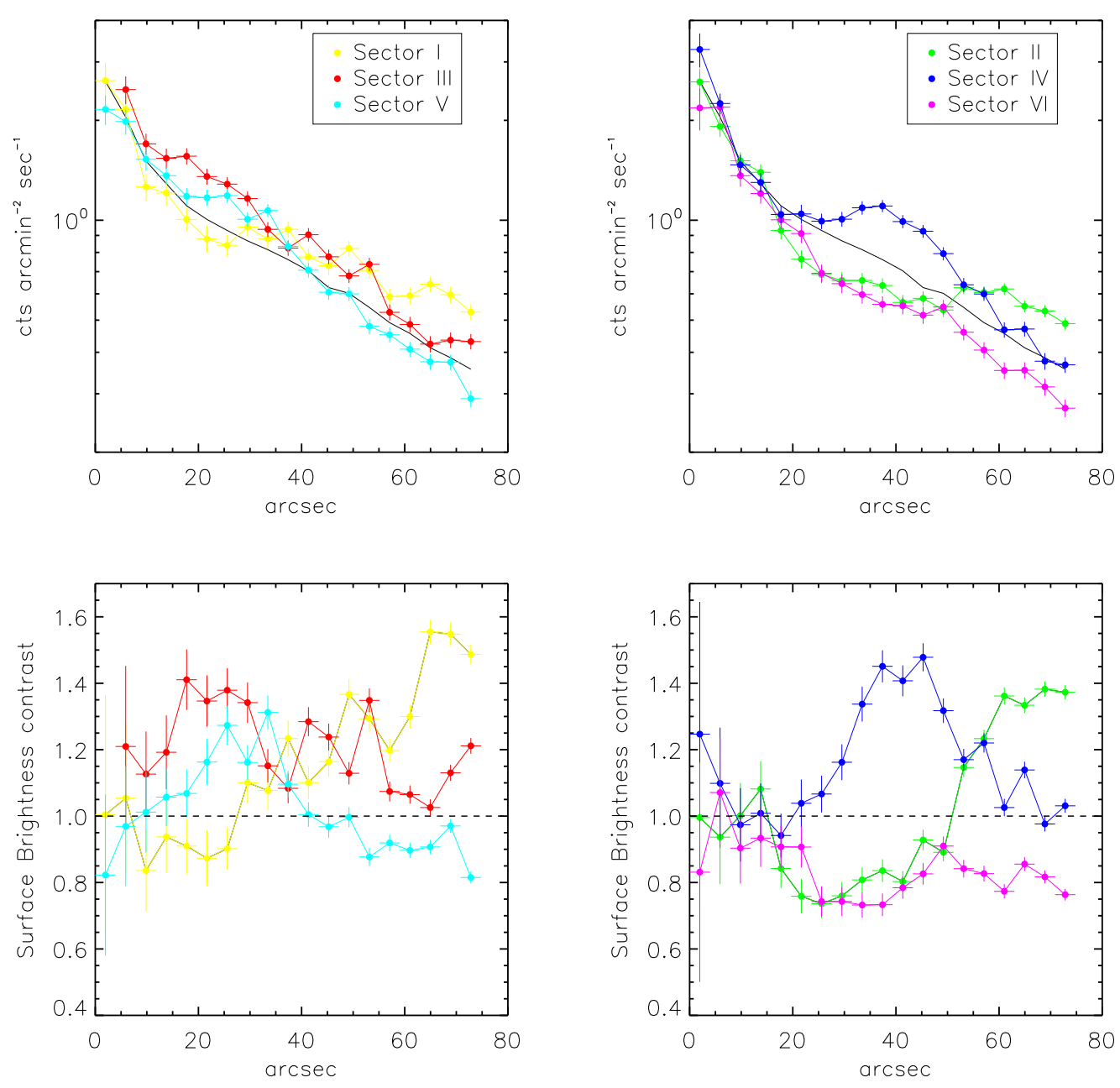

FIG. 3.- Top panel: Surface brightness profiles in selected angular sectors listed in Table 1 and shown in Fig.2. The azimuthally averaged surface brightness profile is plotted as a solid black line in both panels for ease of comparison.

Bottom panel: Ratio of the surface brightness profiles of the above selected angular sectors over the azimuthally averaged one.

TABLE 1

\begin{tabular}{lc}
\hline \multicolumn{1}{c}{ Sector } & PA \\
\hline Sector I & $120-141$ \\
Sector II & $141-182$ \\
Sector III & $182-212$ \\
Sector IV & $212-244$ \\
Sector V & $244-280$ \\
Sector VI & $310-345$ \\
\hline & \\
\hline \multicolumn{2}{l}{ NoTE. - Position an- } \\
gles (measured form the \\
N direction) for the sur- \\
face brightness profiles \\
discussed in the text in \\
§3.1.
\end{tabular}

emission, even brighter than the other two filaments in the range $30^{\prime \prime}-50^{\prime \prime}$ but less extended, with an embedded depression in surface brightness between $10^{\prime \prime}$ and $30^{\prime \prime}$, which stands out as a region of low emission, compared to the surroundings, in Fig.2. However this feature is at the same level of surface brightness of the azimuthally averaged profile. The cavity in sector VI is the real counter-part of the one in sector II. Although it stands out less clearly in the unsharp-mask im- age, it is clearly indicated in the binned images of Fig. 1 and in the striking similarity of the surface brightness profiles within $50^{\prime \prime}$ as shown in Fig.3. The northern cavity stands out less clearly in the unsharp masked image of Fig. 2 because of the dimmer regions surrounding it (the northern arms are not as bright as the southern ones) and because of the sharper drop in surface brightness at $r>50^{\prime \prime}$. There is also evidence of enhanced emission in sector $\mathrm{V}$ in the $20^{\prime \prime}-40^{\prime \prime}$ region, as highlighted also in the unsharp masked image.

\subsection{The inner and outer surface brightness edges}

We now zoom out to investigate the outer regions in the Chandra image, as shown in Fig.4. A sharp edge in the surface brightness on the south-east (PA 120-160) is revealed in this image and further confirmed by a surface brightness profile in the same sector, as compared to a reference profile extracted in the PA 180-315 sector (right panel of Fig.4). Whereas the profile is smooth and with a continuous derivative in the reference sector, the slope of the sector across the cold front is rapidly changing and the edge is at $\sim 31 \mathrm{kpc}$ $\left(170^{\prime \prime}\right)$.

Inspection of the combined MOS image shown in Fig.5 confirms the suggestion of Buote et al. (2003b) of the presence of another surface brightness edge in the north-west di- 

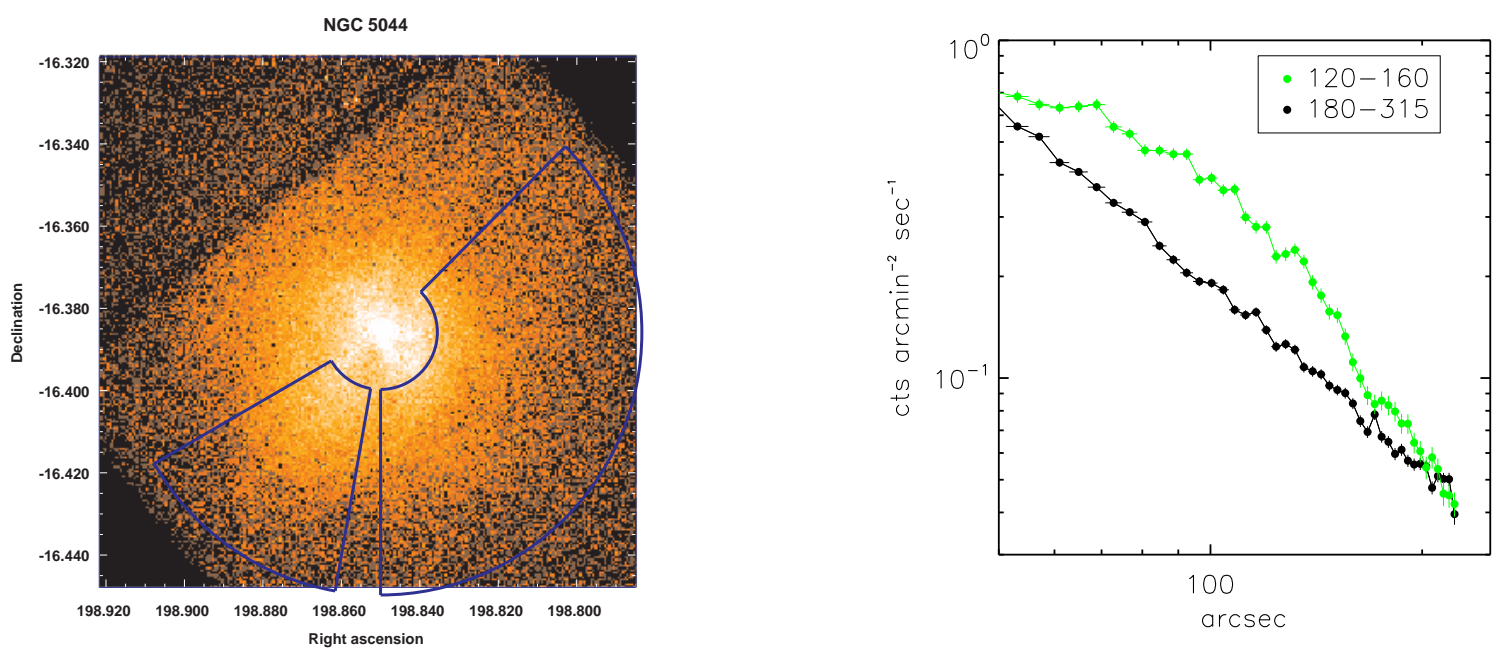

FIG. 4.- Left panel: Over-plotted on the Chandra image are the sector used to extract the surface brightness profile across the cold front and the reference sector used for comparison.

Right panel: Surface brightness profile for the cold front and the reference sector. A sharp drop is visible at $\sim 170^{\prime \prime}$ form the center, i.e. $31 \mathrm{kpc}$.

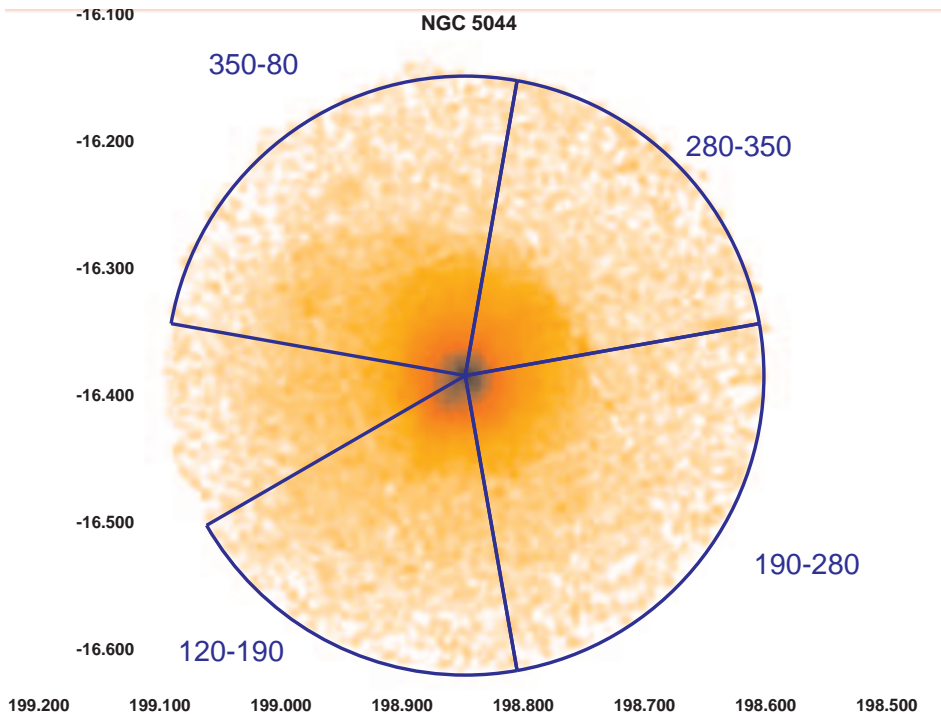

FIG. 5.- Mosaic of the MOS1 and MOS2 images smoothed on a $10^{\prime \prime}$ scale. The image has been divided by the summed exposure maps to correct for exposure variations. Point sources have been removed and filled using the $C I A O$ task dmfilth. Sectors used for the extraction of surface brightness profiles discussed in the text and presented in Fig. 6 are also shown.

rection at $\sim 6^{\prime}$. Plots of the surface brightness profiles across this sector and in other three sectors (one including the inner edge) for comparison are shown in Fig.6. The inner cold front is detected also in the MOS image. More importantly another sharp feature (considering also the XMM PSF at this off-axis angle, $\sim 50$ " $90 \%$ encircled energy fraction at 1.5 $\mathrm{keV}$ ) is clearly revealed at $\sim 350^{\prime \prime}$, i.e. $65 \mathrm{kpc}$ (see right panel of Fig.6). The feature is detected also in the pn data.

\section{SPECTRAL ANALYSIS AND TEMPERATURE MAPS}

Details about the Chandra and XMM spectral extraction and analysis can be found in Humphrey et al. (2006) and Gastaldello et al. (2007b). Here we briefly summarize that for Chandra appropriate count-weighted spectral response matrices were generated for each region using the standard CIAO tasks mkwarf and mkacisrmf and for $X M M$ using the SAS tasks rmfgen and arfgen in extended source mode.
For each spectrum, we estimated the background through local modeling using the method outlined in Humphrey et al. (2006) and Gastaldello et al. (2007b). The spectra were rebinned to ensure a $\mathrm{S} / \mathrm{N}$ of at least 3 and a minimum 20 counts $\mathrm{bin}^{-1}$.

\subsection{The inner $10 \mathrm{kpc}$}

We extracted spectra from the Chandra dataset in a series of regions as depicted in Fig.7, determined using the contour binning technique (Sanders 2006) with a $\mathrm{S} / \mathrm{N}=50$. We fitted the background subtracted spectra with an APEC thermal plasma model (Smith et al. 2001) with the absorbing column density fixed at the Galactic value (Dickey \& Lockman 1990). Solar abundances are in the units of Grevesse \& Sauval (1998). To account for the undetected point-sources we added a $7.3 \mathrm{keV}$ bremsstrahlung component (this model gives a good fit to the spectrum of the detected sources in nearby galaxies, Irwin et al. 2003) for all the regions, because they fall within the twenty-fifth magnitude isophote $\left(\mathrm{D}_{25}\right)$ of the central galaxy NGC 5044.

The temperature map thus obtained is shown in Fig.7. The clearest feature is the cool gas present in the SW (Sector III) arm (cooler than any other region in the map) which is spatially coincident with the $\mathrm{H} \alpha$ and dust filament (Temi et al. 2007b, see Fig.11). The SE arm (Sector I) is hotter. There is presence of cooler gas with respect to the surroundings in the direction of the inner surface brightness edge (i.e. region 26 in Fig.7).

One possible concern about the interpretation of the spectral fits and consequently of the surface brightness features is the suggestion of limited multiphase gas in the temperature structure of NGC 5044, as derived by the preference of two temperature (2T) models over single temperature (1T) models in radial annuli (Buote et al. 2003a). This is further complicated by projection effects, given the fact that we are dealing only with projected spectra in the analysis presented here: a proper deprojection is problematic due to uncertainty in the projection geometry and it is beyond the scope of the present paper. However it is evident in the radial analysis of Buote et al. (2003a) that the cool component clearly dominates in the inner $10 \mathrm{kpc}$ (see their Figure 6) and that a single phase description, modulo projection effects, is an appropri- 

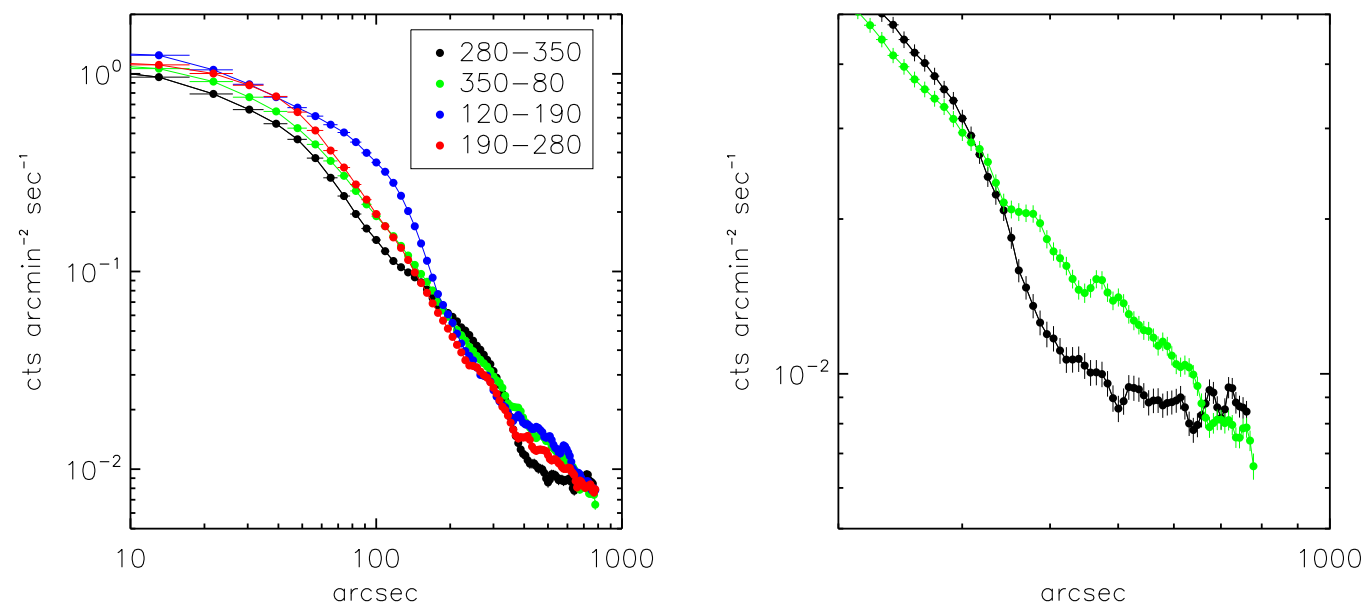

FIG. 6. - Left panel: Surface brightness profiles in selected angular sectors, with PA measured from the N direction. The inner edge can be easily seen, though not as sharp as in the Chandra profile because of the XMM larger PSF.

Right panel: Zoom over the interesting radial region for the outer edge. The characteristic shape of a rapid change in the slope of the profile across the edge can easily be seen in the sector PA 280-350. The profile in the undisturbed sector PA 350-80 is plotted for comparison.

ate description of the data for these inner regions. We investigated fitting 2T models as in Buote et al. (2003a) with the addition of the bremsstrahlung component for the unresolved point sources. The results of the two-dimensional analysis are consistent with the radial analysis of (Buote et al. 2003a) and with the dominance in terms of emission measure of the cool component over the hot one.

To investigate in more detail the nature of the bright soft $\mathrm{X}$-ray filaments we extracted spectra representative of the two southern filaments in two $30^{\prime \prime} \times 45^{\prime \prime}$ rectangular regions at a distance of $30^{\prime \prime}$ from the center (the blue boxes shown in Fig.7); the spectrum taken from the SW filament has a Fe$\mathrm{L}$ feature with lower excitation energy lines more prominent than the spectrum from the SE filament, signature of a lower temperature (see Fig.8). If we fit the two spectra with a 1T model we obtain a temperature difference which is significant at $9 \sigma(\mathrm{kT}=0.65 \pm 0.01 \mathrm{keV}$ for the SE arm and $0.78 \pm 0.01$ $\mathrm{keV}$ for the SW arm). The regions connected to the filaments are cooler compared to all the other regions in the map (by $3.6 \sigma$ compared to the central region \#1, which has the second lowest temperature in the map, $0.70 \pm 0.01$ ). If we try to fit with a $2 \mathrm{~T}$ model the spectrum of the SE arm we obtain only a small decrease in $\chi^{2}\left(\chi^{2} / \mathrm{dof}=57 / 40\right.$ for the $2 \mathrm{~T}$ model compared to $60 / 42$ of the $1 \mathrm{~T}$ model). If we repeat the exercise for the regions of brighter emission surrounding the northern cavity (the cyan boxes shown in Fig.7 encompassing the northern filaments highlighted in Fig. 1) we obtain $0.74 \pm 0.02 \mathrm{keV}$ for the NW filament and $0.82 \pm 0.02 \mathrm{keV}$ for the NE filament.

\subsection{The surface brightness edges}

To investigate the nature of the surface brightness edges we have to determine the temperature across the surface brightness jump. We therefore extracted Chandra spectra for the inner jump together with "control' spectra taken from regions at the same radial distance from the center but at different position angles. We applied the same method to the outer edge when extracting $X M M$ spectra. In Fig.9 we plot the temperature across the edges obtained by fitting a 1T model. In Table 2 we present the results of $1 \mathrm{~T}$ and $2 \mathrm{~T}$ fits for selected regions inside and outside the edges together with control regions at the same radial range but at different position angles.
For the inner edge a clear temperature jump is detected, with the brighter regions inside the edge cooler than the outer part. 2T models are preferred over $1 \mathrm{~T}$ models which points to some degree of projection effects in the temperature determination across the edge, probably caused by the cool gas which is producing the edge. Other regions covering the same range of radii but at different position angles do not show in-fact the same behavior, with the exception to some degree of the region immediately close to the edge. We extracted XMM MOS and pn spectra from the same regions inside and outside the inner edge finding consistent results within the $1 \sigma$ errors for both $1 \mathrm{~T}$ and $2 \mathrm{~T}$ models.

For the outer edge the temperature jump is more subtle and of low statistical significance $(1.6 \sigma)$, but, contrasted with the slightly declining trend of the control regions (we are in the region around the peak of the azimuthally averaged temperature profile), it is consistent with the picture of cooler gas inside the edge.

\section{SYSTEMATIC ERRORS}

This section contains an investigation of possible systematic errors in the data analysis relevant for this paper. A thorough analysis of many of the issues involved has been conducted in Buote et al. (2003a) and Buote et al. (2003b).

\subsection{Image Binning}

The presence of the morphological structures discussed in the paper, in particular the disturbed morphology with filamentary structure of the inner regions, is already evident from the raw Chandra image. The use of the particular binning technique of Sanders (2006) does not introduce any spurious feature. We also used the Weighted Voronoi Tessellation (WVT) binning algorithm of Diehl \& Statler (2006), which is more robust against the introduction of spurious features than adaptive smoothing, as provided for example by the CIAO task csmooth and we found the same features.

\subsection{Plasma Codes}

We compared the results obtained using the APEC code to those obtained using the MEKAL code (Kaastra \& Mewe 1993; Liedahl et al. 1995) to assess the importance of different 

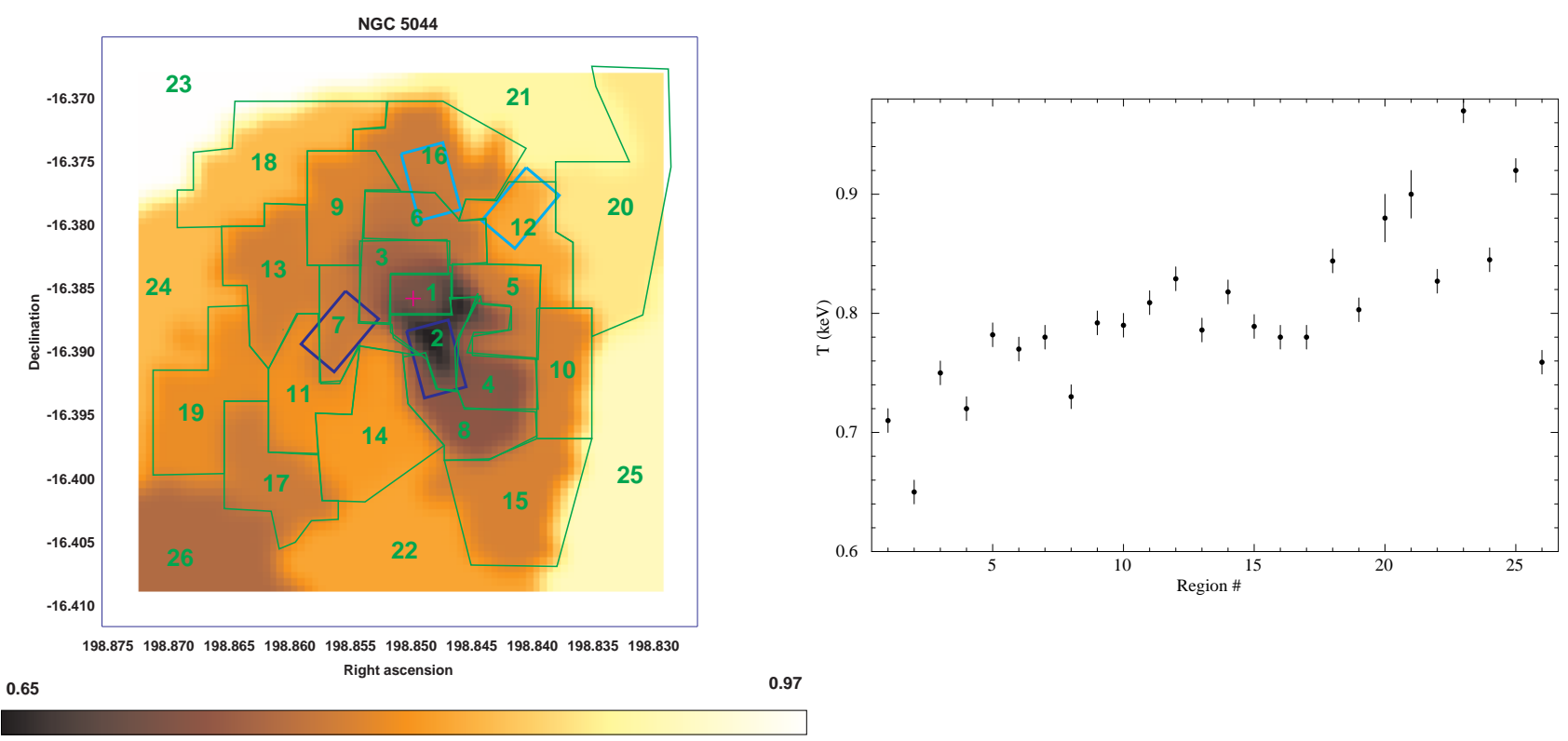

FIG. 7.- Left panel: Temperature map of the core regions of NGC 5044 based on the SN=50 contour binning. The map has been smoothed for better presentation with a Gaussian kernel of $6^{\prime \prime}$. Color bar units are in keV. The magenta cross marks the center of NGC 5044. The spectra presented in Fig.8 and discussed in the text have been extracted from the rectangular regions shown in blue and they encompass the SE and SW filament; the spectra extracted from the cyan boxes and discussed in the text encompass the NE and NW filaments highlighted in Fig.1;. Right panel: Values and $1 \sigma$ error bars for the temperature in each region of the temperature map.

NGC 5044

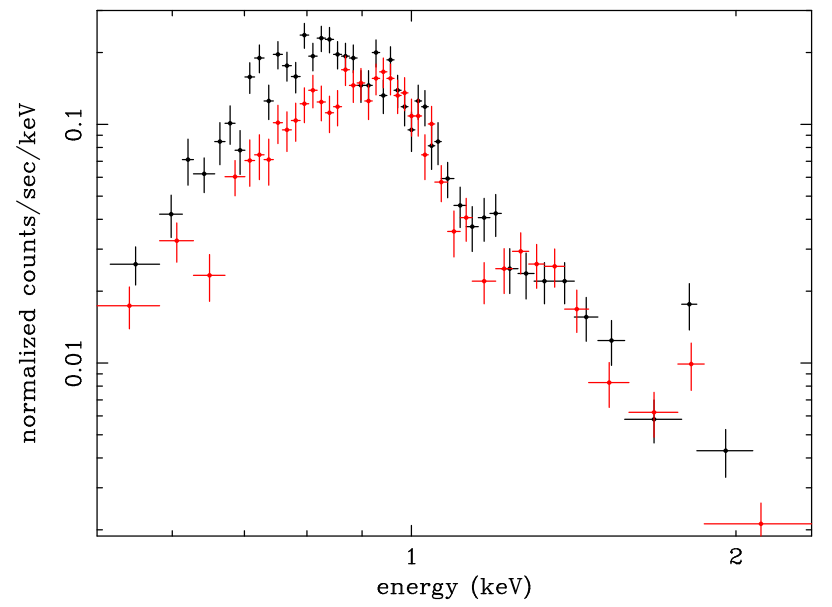

FIG. 8. - The spectra from the rectangular boxes taken respectively from the SW arm (black data points) and the SE arm (red data points). Note the shape of the Fe-L "hump" and the prominence of lower energy lines, signature of lower temperature, in the spectrum of the SW arm.

implementations of the atomic physics and different emission line lists in the plasma codes. We found no qualitative differences between the two codes: the fitted temperatures agree to within 5\% and abundances within 10-20\% (where 1T and 2T models are compared accordingly) and well within their statistical errors.

\subsection{Bandwidth}

We explored the sensitivity of our results to our default lower limit of the bandpass, $E_{\min }=0.5 \mathrm{keV}$. For comparison we performed spectral fits with $E_{\min }=0.4 \mathrm{keV}$ and $E_{\min }=$ $0.7 \mathrm{keV}$. The fitted temperatures are consistent between models; iron abundances are better constrained, in particular for 2T models, when using the larger bandwidth (see Buote et al. 2003b).

\subsection{Variable $N_{\mathrm{H}}$}

We take into account possible deviations for $N_{\mathrm{H}}$ from the value of Dickey \& Lockman (1990) allowing the parameter to vary by $\pm 25 \%$. We found no qualitative differences between the two cases. If we leave $N_{\mathrm{H}}$ free to fit we derive higher values in the core, but still consistent at $1 \sigma$ with the Galactic value in the majority of the regions of Fig.7. All the other spectral parameters are basically unchanged. This is not due to excess absorption but just the exploitation of an additional free parameter by the fitting program to model deviations from a single temperature model.

\subsection{Background}

Since NGC 5044 is sufficiently bright and the temperature determination stems from the measurement of the shape and width of the Fe-L shell which is a prominent feature in the spectra, the fitted temperature values are quite insensitive to errors in the background normalization.

\section{DISCUSSION}

\subsection{The nature of the cavities and filaments in the inner 10 $k p c$}

The X-ray analysis shown in this paper reinforces the scenario proposed for NGC 5044 by Temi et al. (2007b), which we briefly summarize below. In a recent survey of elliptical galaxies observed with the Spitzer telescope Temi et al. (2007a) found spatially extended cold interstellar dust emitting at $70 \mu \mathrm{m}$ around many group-centered X-ray luminous elliptical galaxies. The source of this dust is the dust-rich cores of the central galaxy, not from mergers (Mathews \& Brighenti 2003; Temi et al. 2007a). Since this dust is thought to be in direct contact with the hot, virialized interstellar gas $(k T \sim 1 \mathrm{keV})$, it has a short lifetime $\left(\sim 10^{7} \mathrm{yrs}\right)$ to sputtering destruction by thermal ions. Consequently, this dust is a 

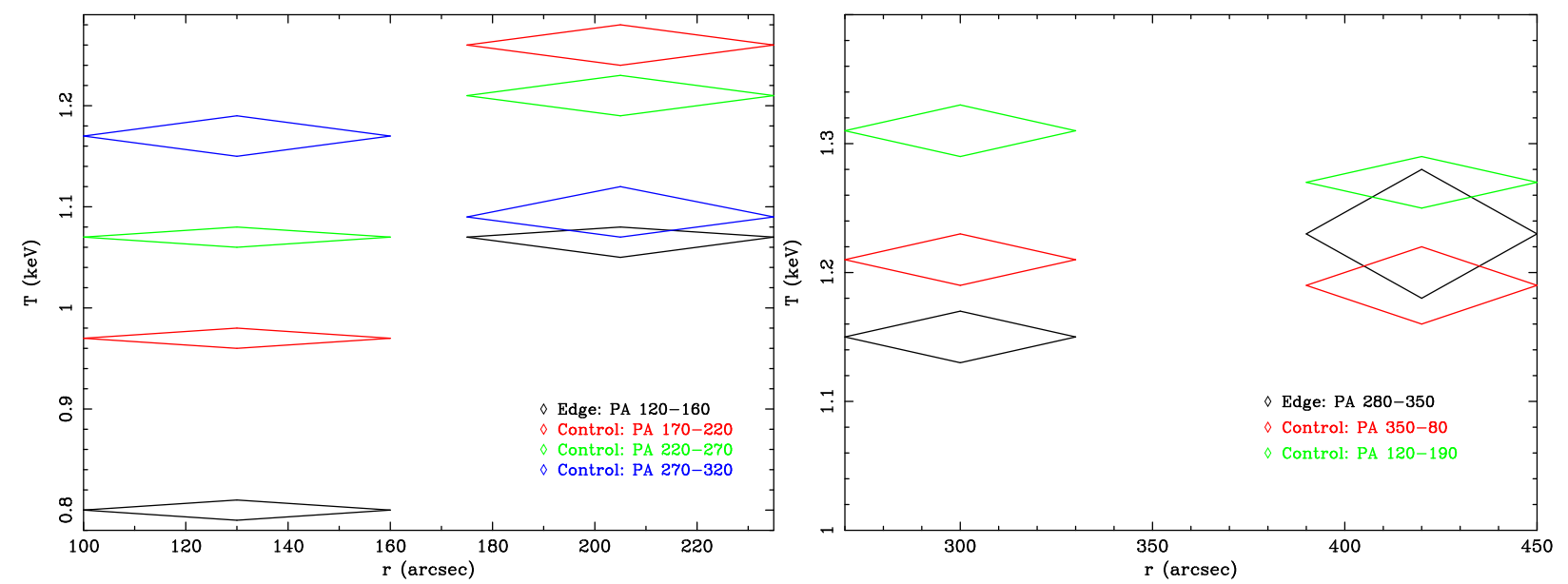

FIG. 9.-Left panel: Values and $1 \sigma$ error bars for the temperature across the inner surface brightness edge and in control sectors.

Right panel: Values and $1 \sigma$ error bars for the temperature across the outer surface brightness edge and in control sectors.

TABLE 2

PARAMETERS FROM THE SPECTRAL FITS ACROSS THE EDGES

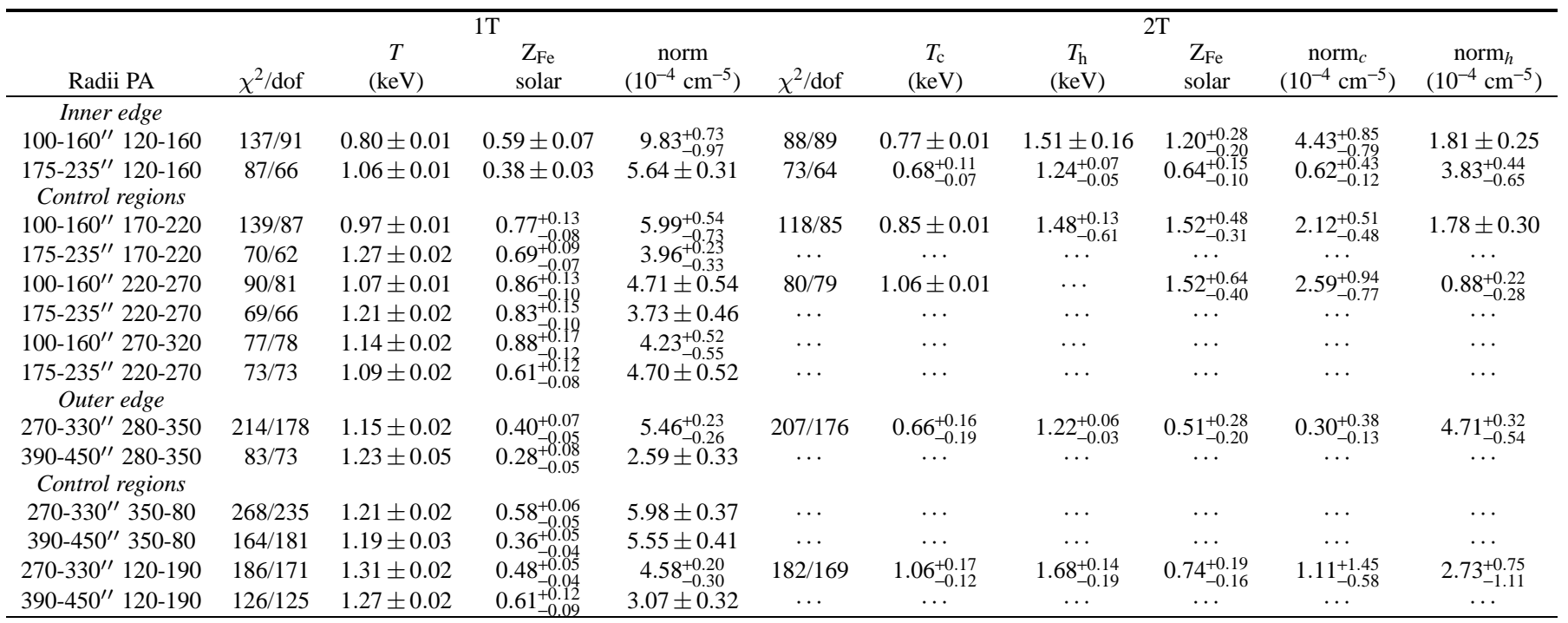

NotE. - Results of $1 \mathrm{~T}$ and $2 \mathrm{~T}$ spectral fits for selected regions as described in the text in $\$ 4.2$. The first and second columns refer to the radial range (in arcseconds) and azimuthal range (PA in degrees) of the fitted sectors discussed in $\$ 4.2$. The norm parameter is the emission measure of the APEC model as defined in Xspec: $10^{-14}\left(\int n_{e} n_{p} d V\right) / 4 \pi D^{2}(1+z)^{2}$ with units $\mathrm{cm}^{-5}$. $T_{\mathrm{c}}$ and $T_{\mathrm{h}}$ refer to the temperature of the cool and hot component of the $2 \mathrm{~T}$ model, whereas norm $_{c}$ and norm $h$ refer to the correspondent emission measures. No entry for $2 \mathrm{~T}$ models means that there was no improvement over a $1 \mathrm{~T}$ model. Regions with no entry for $T_{\mathrm{h}}$ did not significantly constrain that parameter.

spatial tracer of extremely transient events that recently occurred on kpc scales. NGC 5044, together with NGC 4636, has been investigated in more detail in Temi et al. (2007b). NGC 5044 is not only extended at $70 \mu \mathrm{m}$ but also at $8 \mu \mathrm{m}$ in a manner similar to the highly asymmetric $\mathrm{H} \alpha$ optical line emission from warm gas. Current evidence is consistent with the hypothesis that dust has been buoyantly transported from the galactic cores out to several kpc following a feedback heating event. Furthermore, disorganized fragments of optically absorbing dusty gas are visible in $H S T$ images within the central $\sim 100$ parsecs, providing further evidence of a recent central energy release. In particular, the association of interstellar PAH emission (responsible of the $8 \mu \mathrm{m}$ emission) and warm gas $\left(T \sim 10^{4} \mathrm{~K}\right)$ in NGC 5044 indicates that we may be viewing this galaxy at a rare moment immediately following a release of energy near the central black hole.
The Chandra data show the impact of this energy release on the hot X-ray emitting gas which is undergoing short-term gas-dynamical activity. The detection of a pair of X-ray cavities allow us to give an estimate of the amount of energy deposited from the AGN and the time of its release, following a now standard analysis (e.g. Bîrzan et al. 2004; Dunn \& Fabian 2006). For each of the two cavities a size and position was measured, assuming the projected shape is an ellipse measured from the exposure-corrected, un-processed image as depicted in Fig.10. We take as a guide the cavity on the south because of the higher contrast and the surface brightness profiles of Fig.3. The two cavities are at a projected distance $R$ of $33^{\prime \prime}(6.1 \mathrm{kpc})$ from the center of the bubble to the center of NGC 5044, with a projected semi-major axis $a$ of $17^{\prime \prime}$ (3.1 $\mathrm{kpc})$ and a projected semi-minor axis $b$ of $9^{\prime \prime}(1.7 \mathrm{kpc})$. We do not consider here the dip in surface brightness at PA 212-244 
TABLE 3

CAVITIES TIME SCALES AND DERIVED POWERS.

\begin{tabular}{cccccccc}
\hline \hline $\begin{array}{c}p V^{\mathrm{a}} \\
\left(10^{55} \mathrm{erg}\right)\end{array}$ & $\begin{array}{c}t_{\text {sound }} \\
\left(10^{7} \mathrm{yr}\right)\end{array}$ & $\begin{array}{c}t_{\text {buoy }} \mathrm{b} \\
\left(10^{7} \mathrm{yr}\right)\end{array}$ & $\begin{array}{c}t_{\text {ref }} \mathrm{b} \\
\left(10^{7} \mathrm{yr}\right)\end{array}$ & $\begin{array}{c}P_{\text {sound }}{ }^{\mathrm{c}} \\
\left(10^{41} \mathrm{erg} \mathrm{s}^{-1}\right)\end{array}$ & $\begin{array}{c}P_{\text {buoy }}{ }^{\mathrm{c}} \\
\left(10^{41} \mathrm{erg} \mathrm{s}^{-1}\right)\end{array}$ & $\begin{array}{c}P_{\text {ref }} \mathrm{c} \\
\left(10^{41} \mathrm{erg} \mathrm{s}^{-1}\right)\end{array}$ & $\begin{array}{c}L_{X}{ }^{\mathrm{d}} \\
\left(10^{41} \mathrm{erg} \mathrm{s}^{-1}\right)\end{array}$ \\
\hline 4.58 & 1.34 & 1.20 & 3.50 & 1.08 & 1.21 & 0.41 & $32.1 \pm 0.09$ \\
& & 0.69 & 2.02 & & 2.10 & 0.72 & \\
\hline
\end{tabular}

${ }^{\mathrm{a}}$ It refers to the sum of the $p V$ energies of the two cavities

${ }^{b}$ The first value refer to $g$ calculated according to the hydrostatic equilibrium mass estimate whereas the second entry refer to the estimate made using the stellar velocity dispersion of the central galaxy, as described in the text.

${ }^{\mathrm{c}}$ The power is the $p V / t$ work only, with $\gamma /(\gamma-1)$ not accounted for. For a fully relativistic plasma the values for the powers need to be multiplied by 4 .

${ }^{\mathrm{d}}$ Bolometric (0.1-100 keV) X-ray luminosity within a radius of $27 \mathrm{kpc}$, where the cooling time is $3 \mathrm{Gyr}$.

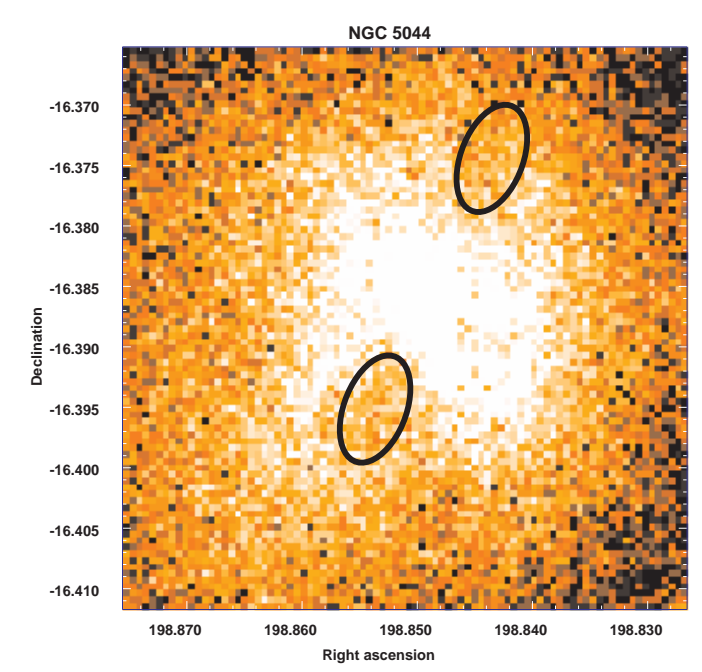

FIG. 10.- Exposure corrected 0.5-5 keV image of NGC 5044. Elliptical regions in black represent the shape and size of the detected X-ray cavities.

because it not a clear deviation from the azimuthally averaged surface brightness profile.

Assuming that the cavities are bubbles completely devoid of gas at the local ambient temperature and taking the pressure and temperature of the gas surrounding the cavities to be the azimuthally averaged values at the projected radius of its center as calculated in the analysis of Gastaldello et al. (2007b), we can calculate the $P \mathrm{~d} V$ work done on the X-ray gas by the AGN in inflating the cavities. The actual third axis $c$ of the ellipsoid is unknown: we assume a prolate shape $(b=c)$. In this case we obtain $p V=2.29 \pm 0.08 \times 10^{55}$ ergs for each cavity (the gas pressure at $6.1 \mathrm{kpc}$ is $2.08 \pm 0.07 \times 10^{-11}$ dyne $\mathrm{cm}^{-2}$ ). Following Bîrzan et al. (2004) we define three different time scales and therefore potential ages for the bubbles: the sonic time scale $t_{c_{s}}=R / c_{s}$, where $c_{s}=445 \pm 3 \mathrm{~km} / \mathrm{s}$ taking $k T=0.77 \pm 0.01$ at $6.1 \mathrm{kpc}$; the buoyancy time scale $t_{\text {buoy }}=R / \sqrt{S C / 2 g V}$ where $V$ is the volume of the bubble, $S$ is the cross section of the bubble and $C=0.75$ is the drag coefficient (Churazov et al. 2001). The gravitational acceleration $g$ can be calculated either as $g=G M(<R) / R^{2}$ using the mass profile derived in Gastaldello et al. (2007b) or, as there can be reasonable doubts about the strict applicability of hydrostatic equilibrium in the inner kpc of NGC 5044 (we are likely underestimating mass and therefore overestimating $t_{\text {buoy }}$, as we argued in Gastaldello et al. 2007b, given also the failure to detect the stellar mass of the central galaxy), we use the stellar velocity dispersion of the central galaxy, under the approximation of an isothermal sphere and calculate $g \approx 2 \sigma^{2} / R$ (Binney \& Tremaine 1987); the time required to refill the displaced volume as the bubble rises upward, $t_{r}=2 \sqrt{r / g}$, again using for $g$ either the X-ray determined mass or the stellar dispersion velocity. The calculated order-of-magnitude estimates for the time scales described above are shown in Table 3 . and they are consistent with the short sputtering lifetime for the extended dust, $\sim 10^{7} \mathrm{yrs}$, discussed in Temi et al. (2007b) and point towards the same episode of AGN feedback.

We can compare estimates of the energy released by the AGN to the radiative losses within the cooling region, here estimated to be the region within a radius where the cooling time is less than 3 Gyr (e.g. Dunn \& Fabian 2006), also tabulated in Table 3 which corresponds to $27 \mathrm{kpc}$. The task of deriving the outburst energy from the observable $p V$ is complicated by various unknowns, like the adiabatic index $\gamma$ of the material inside the bubble $(\gamma=5 / 3$ if thermal or $\gamma=4 / 3$ if non-thermal, see for example the discussion in Mathews \& Brighenti 2008) or the correct hydrodynamic evolution of the bubble expansion, like its initial over-pressure compared to the ICM or the possibility of continuous inflation, to explain the paradoxical result of bubble energy increasing with distance from the center of the gravitational potential (Diehl et al. 2008). Using the commonly adopted recipe of estimating as $4 p V$, with $\gamma=4 / 3$, the energy input arising from the cavities alone, this energy can be at most $\sim 8 \times 10^{41} \mathrm{erg} \mathrm{s}^{-1}$, which falls short to counteract cooling losses.

It is remarkable that a pair of cavities close to the nuclear source are lacking extended high frequency radio emission. This is contrary to what is generally observed in particular in clusters of galaxies. Analysis of VLA observations at 1.56 and $4.9 \mathrm{GHz}$ confirms the presence of just a point source with beam-deconvolved size $<3^{\prime \prime}$ and with a very flat $(\alpha \sim 0)$ spectral index (Giacintucci et al., in preparation). Two other sources sharing the same behavior are NGC 4636 (e.g., Jones et al. 2002) and NGC 4552 (Machacek et al. 2006). However the cavities in these sources have been interpreted as caused by a nuclear outburst which is directly causing shock heating, also because the brighter rims surrounding the cavities are actually hotter than the surrounding medium, rather than displacement by the radio lobes (Jones et al. 2002). Or the cavities could just be related to a previous outburst and might just be ghost cavities, as suggested by O'Sullivan et al. (2005) for NGC 4636 and the same reasoning could apply to NGC 5044. In that case it remains to be explained why the synchrotron emission has faded to undetectable values in these sources and not in cavities with approximately the same ages 

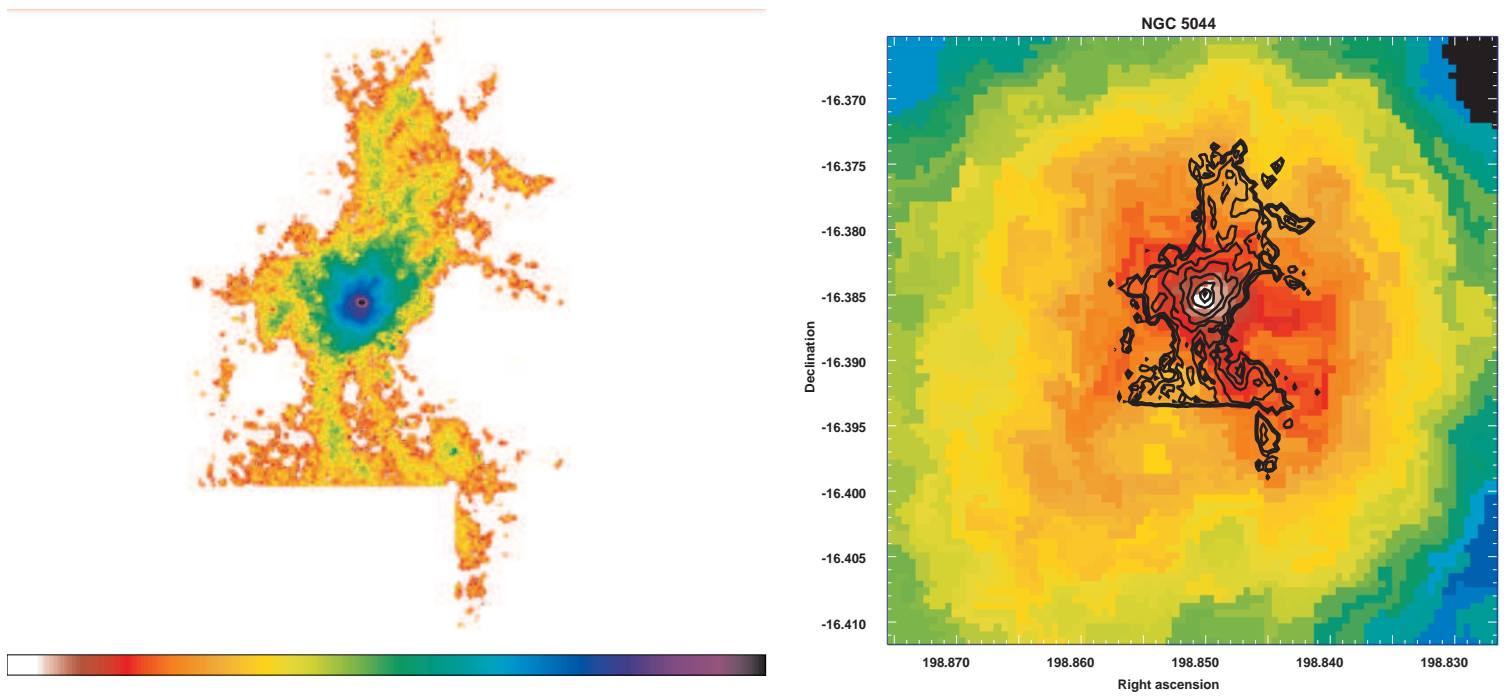

FIG. 11.-Left Panel: $\mathrm{H} \alpha+[\mathrm{N}$ II $]$ map of NGC 5044 taken from Caon et al. (2000). Right Panel: Surface brightness contours of the H $\alpha+[\mathrm{N}$ II $]$ map superimposed on the contour binned image of Fig.1.

in many other sources (e.g., Bîrzan et al. 2004). Other ghost cavity systems with a weak central radio source bearing no obvious relation to the observable cavities which are close to the central galaxy are HCG 62 (Morita et al. 2006) and NGC 741 (Jetha et al. 2008, where only one cavity is present). Sensitive low-frequency radio observations will shed further light on this issue.

The association of dust, $\mathrm{H} \alpha$ and soft $\mathrm{X}$-ray emission showed by Temi et al. (2007b) is also strengthened by the analysis in this paper. The presence of the $\mathrm{N}$ cavity and in particular of the relatively cooler emission in the NW filament (see \$4.1) likely explains the origin of the $\mathrm{N} \mathrm{H} \alpha$ filament. Xray filaments are present at both sides of the cavities, but only the ones with the presence of dust are showing optical emission and cooler X-ray emission (see Fig.11 and the analysis of $\$ 4.1$ ). Temi et al. (2007b) showed that the cospatiality of these features can be explained as the result of dust-assisted cooling in an outflowing plume of hot dusty gas: dust can cool buoyant gas to $10^{4} \mathrm{~K}$, which emits the optical emission lines observed. The warm-gas phase is maintained in thermal equilibrium near $\sim 10^{4} \mathrm{~K}$ by radiative losses and likely a combination of thermal conduction and UV heating from post-AGB stars (see the calculation of Tab.5 in Macchetto et al. 1996, ionization from post-AGB stars could explain only $42 \%$ of the optical line emission in NGC 5044). It is unfortunate that the deeper $\mathrm{H} \alpha$ observation of Caon et al. (2000), compared to the one by Goudfrooij et al. (1994b) used in the comparison of Temi et al. (2007b), is affected by a CCD defect in the southern region co-spatial to the X-ray cavity: it looks like the nebular emission is more extended and can cover also the cavity. Another tantalizing evidence of the association of these three components comes for example from NGC 5846 (Trinchieri \& Goudfrooij 2002, and references therein). Evidence of PAH emission in Spitzer IRS spectra has been found also in NGC 1275 (Johnstone et al. 2007). It has been discussed that AGN feedback can provide some heating (negative feedback), but it can also be responsible for a positive feedback, i.e. for inducing cold gas production (e.g., Pizzolato \& Soker 2005; Revaz et al. 2008). In this scenario AGN feedback itself is responsible for the production of overdense blobs which cool rapidly producing $\mathrm{H} \alpha$ emitting gas, molec- ular gas and star formation. Dust transported from the dusty disks of the central elliptical into the ICM by the episode of AGN feedback acts as a catalyst for the cooling of the gas. It would be therefore interesting for the recent renewed theoretical interest in the generation and survival of the optical line emission filaments (Nipoti \& Binney 2004; Revaz et al. 2008; Pope et al. 2008) to include the neglected dust-assisted cooling in the energy balance equation.

\subsection{The cold fronts and the dynamical state of the group NGC 5044}

The spectral analysis presented in $\$ 4.2$ indicated that the pair of surface brightness discontinuities detected in the Chandra and XMM images are cold fronts. Given the large scale relaxed morphology of NGC 5044 and its rising entropy profile (Gastaldello et al. 2007a) as commonly observed for bright relaxed groups, the source nicely fits in the explanation for the emergence of these features proposed by Ascasibar \& Markevitch (2006) as due to gas sloshing caused by an offaxis merger with a smaller satellite. Another tell-tale sign of this encounter, as also suggested by Ascasibar \& Markevitch (2006), is the presence of a peculiar velocity of the central galaxy, which is often exhibited by central cD galaxies in relaxed clusters (e.g., Oegerle \& Hill 2001). Indeed NGC 5044 is known to have a $\sim 150 \mathrm{~km} / \mathrm{s}$ peculiar velocity with respect to the mean group velocity (Mendel et al. 2008, and references therein). A sub-clump of galaxies statistically significant to the Dressler-Schectman test (Dressler \& Shectman 1988) has been detected in the north-east outskirts of the NGC 5044 (Mendel et al. 2008) which might be the smaller merging sub-group responsible for the gas sloshing and the cold fronts. For a virial mass for NGC 5044 of $3.7 \times 10^{13} \mathrm{M}_{\odot}$ (Gastaldello et al. 2007b), assuming a mass ratio of 5 as in the reference case of Ascasibar \& Markevitch (2006) would imply a mass of $\sim 8 \times 10^{12} \mathrm{M}_{\odot}$ for the satellite. If this candidate sub-clump is real, it would corroborate the proposed scenario and the mechanism proposed by Ascasibar \& Markevitch (2006) for the formation of cold fronts (see also the case of the cluster A 496, Dupke et al. 2007)

Surface brightness discontinuities as cold fronts have so far been discussed in many merging elliptical galaxies and groups 
(e.g., NGC 1404, Machacek et al. 2005). An unusual discontinuity for the group NGC 507, interpreted as an abundance jump, closely related to the expansion of a radio lobe, has been discussed by Kraft et al. (2004). NGC 5044 is to our knowledge the first relaxed group for which cold fronts have been discussed in close similarity to the ubiquitous ones detected in relaxed clusters. Further examples, given enough data quality, are likely to be discovered: suggestions have already been made for objects like MKW 4 and IC 1860 (Gastaldello et al. 2007b).

\section{CONCLUSIONS}

We have presented results of a two-dimensional analysis of the currently available Chandra and XMM data for the bright nearby galaxy group NGC 5044. The results can be summarized as follows:

- A pair of X-ray cavities have been detected, further confirming the recent outburst indicated by the extent and morphology of $\mathrm{H} \alpha$ and dust emission.

- The presence of cooler filamentary X-ray emission cospatial with $\mathrm{H} \alpha$ and dust emission strengthens our previous suggestion that dust-aided cooling could contribute to the $\mathrm{H} \alpha$ emission.

- The presence of a set of two cold fronts together with a peculiar velocity of the central galaxy NGC 5044 suggests a disturbance of an overall relaxed system by an off-axis merger with a smaller satellite.
The detection of such a rich phenomenology has been possible due to NGC 5044 being bright and nearby, much like on bigger mass scales has been possible with M87 and Perseus. As for these objects up-coming deeper X-ray observations with Chandra (David 2007) and XMM (Kaastra 2007) will shed further light on the properties of NGC 5044 and groups of galaxies in general. Multi-wavelength observations, i.e. in $\mathrm{H} \alpha$ and radio bands, are needed for a deeper understanding of the physical processes in the core.

We would like to thank N. Caon for kindly providing the $\mathrm{H} \alpha$ image used in Figure 11 and for useful discussions; S. Giacintucci for sharing her results prior to publication and for useful discussions; P.J. Humphrey for the use of his Chandra data reduction and analysis code; J. Sanders for the use of his contour binning code; L. Zappacosta for a critical reading of the manuscript; the anonymous referee for a carefulr reading and for suggestions that improved the work presented here. We made use of the WVT binning algorithm by Diehl \& Statler (2006), which is a generalization of Cappellari \& Copin's (2003) Voronoi binning algorithm. F.G., F.B. and S.E. acknowledge the financial contribution from contract ASIINAF I/023/05/0 and I/088/06/0. D.A.B. gratefully acknowledges partial support from NASA grant NNG04GE76G, issued through the Office of Space Sciences Long-Term Space Astrophysics Program.

\section{Facilities: CXO, XMM}

\section{REFERENCES}

Allen, S. W., Dunn, R. J. H., Fabian, A. C., Taylor, G. B., \& Reynolds, C. S 2006, MNRAS, 372, 21

Allen, S. W., Edge, A. C., Fabian, A. C., Boehringer, H., Crawford, C. S. Ebeling, H., Johnstone, R. M., Naylor, T., \& Schwarz, R. A. 1992, MNRAS, 259, 67

Ascasibar, Y. \& Markevitch, M. 2006, ApJ, 650, 102

Best, P. N., von der Linden, A., Kauffmann, G., Heckman, T. M., \& Kaiser, C. R. 2007, MNRAS, 379, 894

Binney, J. \& Tremaine, S. 1987, Galactic dynamics (Princeton, NJ, Princeton University Press, 1987, 747 p.)

Bîrzan, L., Rafferty, D. A., McNamara, B. R., Wise, M. W., \& Nulsen, P. E. J. 2004, ApJ, 607, 800

Buote, D. A., Brighenti, F., \& Mathews, W. G. 2004, ApJ, 607, L91

Buote, D. A., Lewis, A. D., Brighenti, F., \& Mathews, W. G. 2003a, ApJ, 594 741

-. 2003b, ApJ, 595, 151

Burns, J. O. 1990, AJ, 99, 14

Caon, N., Macchetto, D., \& Pastoriza, M. 2000, ApJS, 127, 39

Cardiel, N., Gorgas, J., \& Aragon-Salamanca, A. 1998, MNRAS, 298, 977

Churazov, E., Brüggen, M., Kaiser, C. R., Böhringer, H., \& Forman, W. 2001 ApJ, 554, 261

Churazov, E., Sunyaev, R., Forman, W., \& Böhringe r, H. 2002, MNRAS, 332, 729

Clarke, T. E., Sarazin, C. L., Blanton, E. L., Neumann, D. M., \& Kassim, N. E. 2005, ApJ, 625, 748

Conselice, C. J., Gallagher, III, J. S., \& Wyse, R. F. G. 2001, AJ, 122, 2281

Crawford, C. S. 2004, in Clusters of Galaxies: Probes of Cosmological Structure and Galaxy Evolution, ed. J. S. Mulchaey, A. Dressler, \& A. Oemler

Crawford, C. S., Allen, S. W., Ebeling, H., Edge, A. C., \& Fabian, A. C. 1999, MNRAS, 306, 857

Croston, J. H., Hardcastle, M. J., \& Birkinshaw, M. 2005, MNRAS, 357, 279

Croston, J. H., Hardcastle, M. J., Birkinshaw, M., Worrall, D. M., \& Laing, R. A. 2008, MNRAS, 386, 1709

David, L. 2007, in Chandra Proposal, 2448-+

Dickey, J. M. \& Lockman, F. J. 1990, ARA\&A, 28, 215

Diehl, S., Li, H., Fryer, C., \& Rafferty, D. 2008, ArXiv e-prints, 801

Diehl, S. \& Statler, T. S. 2006, MNRAS, 368, 497

Donahue, M. \& Voit, G. M. 2004, in Clusters of Galaxies: Probes of Cosmological Structure and Galaxy Evolution, ed. J. S. Mulchaey, A. Dressler, \& A. Oemler, 143-+

Dressler, A. \& Shectman, S. A. 1988, AJ, 95, 985

Dunn, R. J. H. \& Fabian, A. C. 2006, MNRAS, 373, 959

Dupke, R. \& White, III, R. E. 2003, ApJ, 583, L13
Dupke, R. A., Mirabal, N., Bregman, J. N., \& Evrard, A. E. 2007, ApJ, 668, 781

Edge, A. C. 2001, MNRAS, 328, 762

Fabian, A. C. 1994, ARA\&A, 32, 277

Fabian, A. C., Sanders, J. S., Allen, S. W., Crawford, C. S., Iwasawa, K. Johnstone, R. M., Schmidt, R. W., \& Taylor, G. B. 2003a, MNRAS, 344, L43

Fabian, A. C., Sanders, J. S., Crawford, C. S., Conselice, C. J., Gallagher, J. S., \& Wyse, R. F. G. 2003b, MNRAS, 344, L48

Fabian, A. C., Sanders, J. S., Taylor, G. B., Allen, S. W., Crawford, C. S., Johnstone, R. M., \& Iwasawa, K. 2006, MNRAS, 366, 417

Ferland, G. J., Fabian, A. C., Hatch, N. A., Johnstone, R. M., Porter, R. L., van Hoof, P. A. M., \& Williams, R. J. R. 2008, MNRAS, 386, L72

Finoguenov, A. \& Jones, C. 2001, ApJ, 547, L107

Forman, W., Nulsen, P., Heinz, S., Owen, F., Eilek, J., Vikhlinin, A., Markevitch, M., Kraft, R., Churazov, E., \& Jones, C. 2005, ApJ, 635, 894 Gastaldello, F., Buote, D. A., Humphrey, P. J., Zappacosta, L., Brighenti, F., \& Mathews, W. G. 2007a, in Heating versus Cooling in Galaxies and Clusters of Galaxies, ed. H. Böhringer, G. W. Pratt, A. Finoguenov, \& P. Schuecker, 275-+

Gastaldello, F., Buote, D. A., Humphrey, P. J., Zappacosta, L., Bullock, J. S., Brighenti, F., \& Mathews, W. G. 2007b, ApJ, 669, 158

Ghizzardi, S., Molendi, S., Rossetti, M., \& Leccardi, A. 2007, in Heating versus Cooling in Galaxies and Clusters of Galaxies, ed. H. Böhringer, G. W. Pratt, A. Finoguenov, \& P. Schuecker, 33-+

Goudfrooij, P., Hansen, L., Jorgensen, H. E., \& Norgaard-Nielsen, H. U. 1994a, A\&AS, 105, 341

Goudfrooij, P., Hansen, L., Jorgensen, H. E., Norgaard-Nielsen, H. U., de Jong, T., \& van den Hoek, L. B. 1994b, A\&AS, 104, 179

Grevesse, N. \& Sauval, A. J. 1998, Space Science Reviews, 85, 161

Hatch, N. A., Crawford, C. S., Johnstone, R. M., \& Fabian, A. C. 2006 MNRAS, 367, 433

Humphrey, P. J., Buote, D. A., Gastaldello, F., Zappacosta, L., Bullock, J. S.

Brighenti, F., \& Mathews, W. G. 2006, ApJ, 646, 899

Irwin, J. A., Athey, A. E., \& Bregman, J. N. 2003, ApJ, 587, 356

Jaffe, W., Bremer, M. N., \& Baker, K. 2005, MNRAS, 360, 748

Jetha, N. N., Hardcastle, M. J., Babul, A., O’Sullivan, E., Ponman, T. J., Raychaudhury, S., \& Vrtilek, J. 2008, MNRAS, 384, 1344

Jetha, N. N., Ponman, T. J., Hardcastle, M. J., \& Croston, J. H. 2007, MNRAS, 376, 193

Johnstone, R. M. \& Fabian, A. C. 1988, MNRAS, 233, 581

Johnstone, R. M., Hatch, N. A., Ferland, G. J., Fabian, A. C., Crawford, C. S., \& Wilman, R. J. 2007, MNRAS, 382, 1246 
Jones, C., Forman, W., Vikhlinin, A., Markevitch, M., David, L., Warmflash, A., Murray, S., \& Nulsen, P. E. J. 2002, ApJ, 567, L115

Kaastra, J. 2007, in XMM-Newton Proposal ID \#05546802, 141-+

Kaastra, J. S. \& Mewe, R. 1993, A\&AS, 97, 443

Kraft, R. P., Forman, W. R., Churazov, E., Laslo, N., Jones, C., Markevitch, M., Murray, S. S., \& Vikhlinin, A. 2004, ApJ, 601, 221

Liedahl, D. A., Osterheld, A. L., \& Goldstein, W. H. 1995, ApJ, 438, L115

Macchetto, F., Pastoriza, M., Caon, N., Sparks, W. B., Giavalisco, M., Bender, R., \& Capaccioli, M. 1996, A\&AS, 120, 463

Machacek, M., Dosaj, A., Forman, W., Jones, C., Markevitch, M., Vikhlinin, A., Warmflash, A., \& Kraft, R. 2005, ApJ, 621, 663

Machacek, M., Nulsen, P. E. J., Jones, C., \& Forman, W. R. 2006, ApJ, 648, 947

Markevitch, M., Ponman, T. J., Nulsen, P. E. J., Bautz, M. W., Burke, D. J., David, L. P., Davis, D., Donnelly, R. H., Forman, W. R., Jones, C., Kaastra

J., Kellogg, E., Kim, D.-W., Kolodziejczak, J., Mazzotta, P., Pagliaro, A. Patel, S., Van Speybroeck, L., Vikhlinin, A., Vrtilek, J., Wise, M., \& Zhao, P. 2000, ApJ, 541, 542

Markevitch, M., Vikhlinin, A., \& Forman, W. R. 2003, in Astronomical Society of the Pacific Conference Series, Vol. 301, Astronomical Society of the Pacific Conference Series, ed. S. Bowyer \& C.-Y. Hwang, 37-+

Mathews, W. G. \& Brighenti, F. 2003, ApJ, 590, L5

- 2008, ArXiv e-prints, 805

Mazzotta, P., Markevitch, M., Vikhlinin, A., Forman, W. R., David, L. P., \& VanSpeybroeck, L. 2001, ApJ, 555, 205

McNamara, B. R. 2004, in The Riddle of Cooling Flows in Galaxies and Clusters of galaxies, ed. T. Reiprich, J. Kempner, \& N. Soker, 177-+

McNamara, B. R. \& Nulsen, P. 2007, Annual Review of Astronomy and Astrophysics, 45

Mendel, J. T., Proctor, R. N., Forbes, D. A., \& Brough, S. 2008, MNRAS, 389,749

Morita, U., Ishisaki, Y., Yamasaki, N. Y., Ota, N., Kawano, N., Fukazawa, Y., \& Ohashi, T. 2006, PASJ, 58, 719
Motl, P. M., Burns, J. O., Loken, C., Norman, M. L. ., \& Bryan, G. 2004, ApJ, 606, 635

Narayan, R. \& Medvedev, M. V. 2001, ApJ, 562, L129

Nipoti, C. \& Binney, J. 2004, MNRAS, 349, 1509

Oegerle, W. R. \& Hill, J. M. 2001, AJ, 122, 2858

Osmond, J. P. F. \& Ponman, T. J. 2004, MNRAS, 350, 1511

O’Sullivan, E., Vrtilek, J. M., \& Kempner, J. C. 2005, ApJ, 624, L77

Peterson, J. R. \& Fabian, A. C. 2006, Phys. Rep., 427, 1

Pizzolato, F. \& Soker, N. 2005, ApJ, 632, 821

Pope, E., Pittard, J., Hartquist, T., \& Falle, S. 2008, ArXiv e-prints, 801

Rasmussen, J. \& Ponman, T. J. 2007, MNRAS, 380, 1554

Reiprich, T. H. \& Böhringer, H. 2002, ApJ, 567, 716

Revaz, Y., Combes, F., \& Salomé, P. 2008, A\&A, 477, L33

Salomé, P. \& Combes, F. 2003, A\&A, 412, 657

Sanders, J. S. 2006, MNRAS, 371, 829

Sanderson, A. J. R., Ponman, T. J., \& O’Sullivan, E. 2006, MNRAS, 372 1496

Silk, J., Djorgovski, S., Wyse, R. F. G., \& Bruzual A., G. 1986, ApJ, 307, 415 Smith, R. K., Brickhouse, N. S., Liedahl, D. A., \& Raymond, J. C. 2001, ApJ, 556, L91

Sparks, W. B., Donahue, M., Jordán, A., Ferrarese, L., \& Côté, P. 2004, ApJ, 607, 294

Sparks, W. B., Macchetto, F., \& Golombek, D. 1989, ApJ, 345, 153

Temi, P., Brighenti, F., \& Mathews, W. G. 2007a, ApJ, 660, 1215

-. 2007b, ApJ, 666, 222

Trinchieri, G. \& Goudfrooij, P. 2002, A\&A, 386, 472

Vikhlinin, A., Markevitch, M., \& Murray, S. S. 2001, ApJ, 551, 160

Voigt, L. M. \& Fabian, A. C. 2004, MNRAS, 347, 1130

Vrtilek, J. M., Grego, L., David, L. P., Ponman, T. J., Forman, W., Jones, C. \& Harris, D. E. 2002, APS Meeting Abstracts, 17107

Wilman, R. J., Edge, A. C., \& Swinbank, A. M. 2006, MNRAS, 371, 93 